\title{
THE EFFICACY OF FRACTIONAL CO2 LASER WHEN COMBINED WITH PLATELET-RICH FIBRIN MEMBRANE FOR FACIAL REJUVENATION
}

\section{Dermatology}

Vasim F Chauhan

Third Year Resident, Department of Dermatology, GMERS Medical College \& Hospital, Sola, Ahmedabad.

Krina B Patel*

Professor \& Head, Department of Dermatology, GMERS Medical College \& Hospital, Sola, Ahmedabad. *Corresponding Author

Nitin S Vora

Professor, Department of Dermatology, GMERS Medical College \& Hospital, Sola, Ahmedabad.

\section{ABSTRACT}

Background: Facial rejuvenation is different from other aesthetic procedures in that it directly restores a younger appearance in patients. Demand for a youthful and attractive appearance has increased recently and mass media have promoted the desire for beauty. Fractional-CO2 laser one of the most utilised modality for this purpose nowadays but there are few drawbacks of this method which can be reduced and efficacy can be increased by the addition of autologous blood components.

Objectives: We evaluated the synergistic efficacy and safety of combined use of fractional-CO2 laser and topical application platelet-rich fibrin membrane for facial rejuvenation.

Methodology: Total of 12 patients (M:5, F:7) was included in the study. All patients were treated once with fractional-CO2 laser followed by topical application of platelet-rich fibrin membrane application over face for 30 minutes.Patients were evaluated at baseline, one, four, and eight weeks after the session.

Result: PRF membrane when applied after fractional-CO2 laser showed additive effects on facial rejuvenation with lesser downtime.

\section{KEYWORDS}

\author{
Laser, Rejuvenation, Platelet-rich Fibrin
}

\section{INTRODUCTION}

Smooth, wrinkle-free skin is associated with attractiveness, youthfulness, and positive mental health, while rhytids hurt one's perceived appearance and self-esteem.

The severity of the ageing process, involvement and the character of the skin varies among patients. Individual factors, such as the patient's expectations, the recovery time required until the patient can return to daily social activities, and the patient's economic status also influence the choice of rejuvenation method. Hence, one of the greatest difficulties a dermatologist face in approaching facial rejuvenation is individual diversity, in light of the plethora of technical approaches available for improving the appearance of ageing faces with minimal downtime and lesser cost. ${ }^{[1}$

Carbon dioxide (CO2) laser skin resurfacing has been a mainstay of facial rejuvenation since its introduction in the mid-1990s. Recently, a new generation of fractional-CO2 (photothermolysis) lasers has been introduced which creates microscopic thermal wounds that ablate the epidermis and dermis within very tiny zones; adjacent to these areas, the epidermis and dermis are spared. This microablative process of laser skin resurfacing has proven safe and effective for facial rejuvenation, capable of improving wrinkles, acne scars, and other types of atrophic scars and benign pigmented lesions associated with elastotic, sun-damaged skin. Fractional- $\mathrm{CO}_{2}$ laser has now been considered as the gold standard for skin rejuvenation.

It often requires multiple therapy sessions which will be a kind of psychological and economic burden for the patients. Besides that, the fractional- $\mathrm{CO}_{2}$ laser is still reported to have a variety of complications, such as post-inflammatory hyperpigmentation, prolonged erythema, skin swelling, infection, and scarring. ${ }^{[3]}$

To overcome these drawbacks while increasing the effect of fractional$\mathrm{CO} 2$ with fewer sessions, is one hot topic in the dermatological research field.

In this sense, platelet-rich fibrin (PRF) membrane ${ }^{[4]}$, achieved from autologous blood, appears as a natural and satisfactory alternative with favourable results and low risks.

PRF was first used in 2001 by Choukroun et al. ${ }^{[5]}$, specifically in oral and maxillofacial surgery, and is currently considered as a new generation of platelet concentrate. It consists of a matrix of autologous fibrin and has several advantages over PRP, including easier preparation and not requiring chemical manipulation of the blood, which makes it strictly an autologous preparation. ${ }^{[6]}$

Platelets containing alpha-granules are large macromolecules that constitute $15 \%$ of the total platelet volume. They contain plateletspecific and non-platelet-specific proteins (fibrinogen, fibronectin, thrombospondin, growth factors, etc.). Growth factors stimulate and attract stem cells to the site of injury, promoting cell mitosis and inducing angiogenesis. Such growth factors when trapped in the fibrin matrix, can lead to faster and prolonged healing of the tissue. ${ }^{[7}$ Furthermore, fibrin is a bridging molecule that allows a series of cell interactions and supplies a provisional matrix in which cells may proliferate, organize, and carry out their functions, mainly, in sites that suffered injury or inflammation. ${ }^{[8]}$

\section{MATERIALAND METHOD}

In this study, we enrolled twelve subjects with written informed consent. Five male and seven female patients with Fitzpatrick phototypes III and IV were enrolled. Patients were aged between 25-34 years (mean age- 29.2 years). Active skin infections, warts, recurrent herpes infections, history of keloid or hypertrophic scars, positive serology of HIV, HbsAg or VDRL test were our exclusion criteria. Patients having unrealistic expectations, pregnant, diabetic or patients taking corticosteroids, antiplatelet or anticoagulant drugs were also excluded.

\section{Procedure:}

Patients were primed with topical tretinoin gel and sunscreen cream for 4 weeks before the session. Complete blood count with serology was done including serum HBsAg, HIV and VDRL test. Pre-treatment digital photographs including both sides and front of the face were taken under consistent background, position and proper lighting.

Topical application of anaesthetic cream (eutectic mixture of lidocaine and prilocaine) was applied for 30 minutes before treatment and then completely removed and face was cleaned with saline and acetone solution.

For PRF membrane, $20 \mathrm{ml}$ autologous venous blood was withdrawn from the patient, taken into the plain sterile vacutainer without anticoagulant, centrifuged for $3000 \mathrm{rpm}$ for 5 minutes. Yellowishwhite, semitranslucent and semiliquid supernatant was spread over three sets of pre-prepared paraffin impregnated gauge over the sterile dish. It becomes more yellowish with a gel-like consistency and settles on the gauge-pieces to form a membrane.

Meanwhile, a fractional- $\mathrm{CO}_{2}$ laser $\left(\right.$ Dolphin $^{\mathrm{TM}}$ Laser Technology, 
Ahmedabad, India) was used for the treatment of both the cheeks and forehead. First Pass with $18 \mathrm{w}$ energy-power, $2.0 \mathrm{~mm}$ distance, $2 \mathrm{~ms}$ duration and $36 \mathrm{~mJ}$ was done and ice-pack was applied for 5 minutes. Following the second pass was given with $18 \mathrm{w}$ energy-power, $0.5 \mathrm{~mm}$ distance, $0.5 \mathrm{~ms}$ duration, $10 \mathrm{w}$ power and $5 \mathrm{~mJ}$ energy.

Prepared PRF-membranes (one for the forehead and one each for cheeks) were spread over this laser-treated areas and kept for 30 minutes.

Proper cleaning and application of sunscreen cream were done in the aftermath. Patients were advised for the daily sun-protection for further 8 weeks. Patients were followed up for the following one, four and eight weeks. At each follow-up visit, photographs were taken and apparent improvement, the presence or absence of erythema, infection and dyschromia were noted.

The outcome assessments of the clinical improvement using a grading scale of grade- $1 \leq 25 \%$, grade- $2=26-50 \%$, grade- $3=51-75 \%$, grade$4=75-90 \%$ and grade- $5=91-100 \%$ improvement. It was achieved by subjective assessment of fine wrinkles, coarse wrinkles, roughness, mottled hyperpigmentation, laxity, skin tone, texture and patient satisfaction.

\section{OBSERVATIONAND ANALYSIS}

Each patient tolerated the procedure well. Out of 12 patients, two patients (17\%) achieved grade 4 improvement, while six $(50 \%)$ had grade 3 and the remaining four patients $(33 \%)$ achieved grade 2 improvement.

Each patient earned more than 50\% improvement. Mean improvement was $74.83 \%$ (SD $12.55 \%$ ) with this single session therapy.

There was no incidence of infection, hypopigmentation or scarring. Post-procedural erythema and burning pain were common and transient.

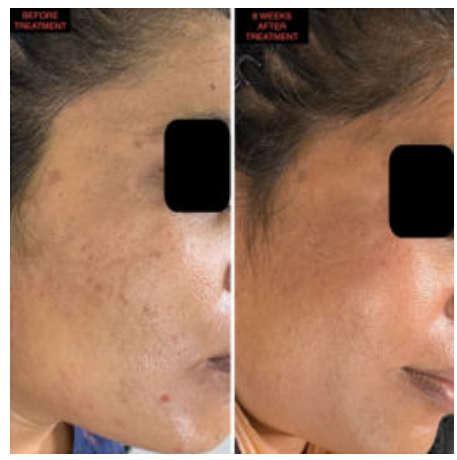

Figure 1

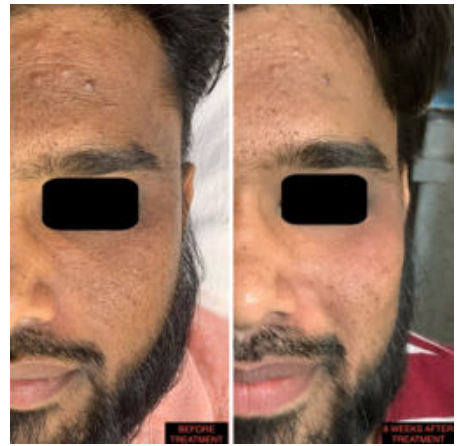

Figure 2

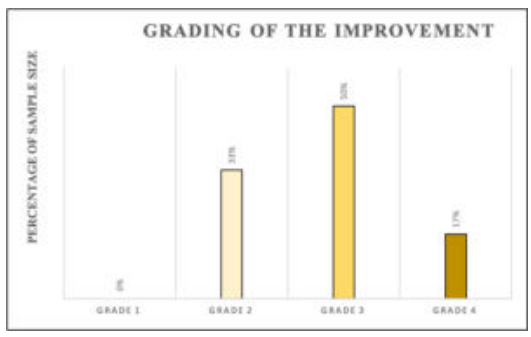

DISCUSSION

The fractional $\mathrm{CO} 2$ laser uses thermal-energy leaving behind intervening zones or normal skin for rapid regeneration. Due to such heat production, can lead to inflammation, oedema, erythema which may cause hypo- or hyperpigmentation or infections. Though its unmatched performance for facial rejuvenation, these flaws can lead to dissatisfaction of the patients. ${ }^{[9]}$

To diminish such blemish, we used PRF, a supraphysiologic concentrate of autologous platelets which is full of growth factors having a great capacity to support cell migrations and healing processes. Dohan et al. (2006) stated that PRF was a completely usable healing concentrate. ${ }^{[7]}$

Hom et al. (2007) in a study on the healing effects of autologous platelet gel (APG) on acute human skin wounds concluded that APG not only enhanced wound closure but also increased the wound healing velocity. $^{[10]}$

PRP, a first-generation plasma concentrate, contains $95 \%$ platelets, $4 \%$ $\mathrm{RBC}$, and $1 \% \mathrm{WBC}$. PRP is also rich in growth factors. Arsiwala el al (2020) compared the efficacy of fractional CO2 laser when combined with platelet-rich plasma (PRP) for facial scars and concluded that considerable reduction in pain and hyperpigmentation was observed with higher efficacy and more patient satisfaction in a patient treated with the laser with PRP. ${ }^{[1]}$

PRP requires anticoagulants and more blood with more speed for centrifugation to achieve adequate amount. Higher spin produces higher energy which can damage growth-factors. Due to the various advantages of PRF over PRP and the ease of technique, PRF is preferred over the PRP which is a simple approach of concentrating platelets without using anticoagulants and it initiates a more rapid and complete healing process. ${ }^{[12]}$

A study by Jain et $\mathrm{al}^{[13],}$ revealed that maximum response seen in two patients was grade 4 improvement treated with a CO2 laser. While in this study, the maximum response was grade 5 improvement when combined laser with PRF.

Basak Yalici-Armagan et al study concluded that rejuvenation with the fractional ablative carbon dioxide laser decreases the symptoms of facial ageing mildly $n$ more than half of the patients ${ }^{[14]}$. This study revealed that more than $70 \%$ improvement was seen in two-fourth of the patients when the laser is combined with PRF for facial rejuvenation.

Jie Zhu el al studied the efficacy of fractional CO2 with type A Botulinum toxin for facial rejuvenation in a split-face study and disclosed that botulinum toxin enhances the result of the fractional CO2 laser. ${ }^{[15]}$

On the other side PRF, inexpensive and much safer than botulinum toxin, can provide similar enhancement with a laser.

Further study with larger sample size and randomization control trials are required to reinforce these pieces of evidence.

\section{CONCLUSION}

Autologous PRF membrane not only enhances the effect of fractional $\mathrm{CO} 2$ laser but it also reduces possible adverse effects of the laser when applied topically after the procedure.

\section{REFERENCES:}

Kim BJ, Choi JH, Lee Y. Development of Facial Rejuvenation Procedures: Thirty Years of Clinical Experience with Face Lifts. Arch Plast Surg. 2015;42(5):521-531.

2. Saedi N, Jalian HR. Fractionation: past, present, future. Semin Cutan Med Surg. 2012 Jun;31(2):105-9

3. $\mathrm{Oh} \mathrm{BH}$, Hwang YJ, Lee YW. Skin characteristics after fractional photothermolysis. An Dermatol. 2011 Nov; 23(4):448-54.

4. Jagati A, Chaudhary RG. Preparation of Platelet-rich Fibrin Membrane over Scaffold of Collagen Sheet, its Advantages over Compression Method: A Novel and Simple Technique. J Cutan Aesthet Surg. 2019;12(3):174-178, doi:10.4103/JCAS. JCAS $153 \_18$

5. Choukroun J, Adda F. Une opportunité en paro-implantologie: le PRF. Implantodontie. 2000;42:55-62.

6. Kiran NK, Mukunda KS. Platelet concentrates: A promising innovation in dentistry. J Dent Sci Res. 2011;2:50-61.

7. Dohan DM, Choukroun J. Platelet-rich fibrin (PRF): a second-generation platelet concentrate. Part II: platelet-related biologic features. Oral Surg Oral Med Oral Pathol Oral Radiol Endod. 2006:101:e45-50.

8. Laurens N, Koolwijk P.Fibrin structure and wound healing. J Thromb Haemost. Laurens N, Koo 
9. Gawdat HI, Hegazy RA.Autologous platelet rich plasma: topical versus intradermal after fractional ablative carbon dioxide laser treatment of atrophic acne scars. Dermatol Surg 2014;40:152-61

10. Hom DB, Linzie BM, Huang TC. The healing effects of autologous platelet gel on acute human skin wounds. Arch Facial Plast Surg. 2007 May-Jun;9(3):174-83.

11. Desai CB, Mahindra UR. Use of Platelet-Rich Fibrin over Skin Wounds: Modified Secondary Intention Healing. J Cutan Aesthet Surg. 2013 Jan; 6(1):35-37.

12. Nazneen A. A comparative study to assess the efficacy of fractional carbon dioxide laser and a combination of fractional carbon dioxide laser with topical autologous plateletrich plasma in post-acne atrophic scars. J Cutan Aesthet Surg. 2020 Mar; 13(1):11-17

13. Jain VK. $\mathrm{Co}_{2}$ laser resurfacing for facial rhytides. J Cutan Aesthet Surg 2008;1:19-20

14. Yalici-Armagan B. Fractional carbon dioxide laser for facial rejuvenation: A prospective study to evaluate the efficacy and the safety. J Cosmet Laser Ther.2018 Feb;20(1):2-8

15. Jie Z. The Efficacy and Safety of Fractional $\mathrm{CO}_{2}$ Laser Combined with Topical Type A Botulinum Toxin for Facial Rejuvenation: A Randomized Controlled Split-Face Study. Biomed Res Int. 2016;2016:3853754. 